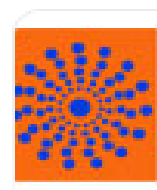

JOURNAL OF BEHAVIOR, HEALTH \& SOCIAL ISSUI

imis

Jiménez-López, Leticia María; Jurado Cárdenas, Samuel ANSIEDAD SOCIAL CONSUMO RIESGOSO DE ALCOHOL EN ADOLESCENTES MEXICANOS Journal of Behavior, Health \& Social Issues, vol. 6, núm. 1, mayo-octubre, 2014, pp. 37-50 Asociación Mexicana de Comportamiento y Salud, A. C. Distrito Federal, México
Journal of Behavior, Health \& Social Issues

ISSN: 2007-0780

jcpedro@unam.mx

Asociación Mexicana de Comportamiento y

Salud, A. C.

México

Disponible en: http://www.redalyc.org/articulo.oa?id=282231041003

Cómo citar el artículo

- Número completo

- Más información del artículo

- Página de la revista en redalyc.org 


\section{ANSIEDAD SOCIAL CONSUMO RIESGOSO DE ALCOHOL EN ADOLESCENTES MEXICANOS}

\section{SOCIAL ANXIETY AND ALCOHOL RISKY DRINKING AMONG MEXICAN ADOLESCENTS}

\author{
Leticia María Jiménez-López \\ Centro Interdisciplinario de Ciencias de la Salud. \\ Unidad Santo Tomás, Instituto Politécnico Nacional. \\ Ciudad de México, Distrito Federal. México

\section{Dr. Samuel Jurado Cárdenas} \\ Facultad de Psicología. Universidad Nacional \\ Autónoma de México. Ciudad de México, Distrito \\ Federal. México.
}

\begin{abstract}
Recibido: Julio 18, 2013
Revisado: Septiembre 22, 2013

Aceptado: Octubre 28, 2013

Los autores agradecen al Instituto Politécnico Nacional y a la Secretaría de Educación Pública, por las facilidades prestadas para la realización del presente trabajo. El presente trabajo fue realizado en la Ciudad de México, D.F., como parte de un estudio más amplio, que constituyó la tesis doctoral de la primera autora (LMJL), bajo la dirección del segundo (SJC). Cualquier comunicación dirigirla a: Leticia María Jiménez López. Departamento de Posgrado. Centro Interdisciplinario de Ciencias de la Salud, Unidad Santo Tomás, Instituto Politécnico Nacional. Av. de los Maestros S/N Col. Santo Tomás, Delegación Miguel Hidalgo, C.P.11340. México, D. F. Tel. 57296000- Ext 63461. Cel. 5545309143. correo electrónico: leticiamariađmsn.com
\end{abstract}

Resumen

Gradualmente se incrementa la investigación acerca de la fobia social, argumentando asociación entre ésta y diversas medidas de disfuncionalidad. Se obtuvieron datos sociodemográficos, mediciones de ansiedad social y de consumo de alcohol, de 3164 adolescentes mexicanos escolarizados voluntarios (hombres y mujeres) entre 12 y 18 años de edad ( $\bar{x}=14.7, D E 1.7$ ), en distinto turno escolar (matutino o vesperino) y con registro de sus actividades extracurriculares (realizar o no actividades deportivas, artísticas y tener o no una relación de noviazgo). Se formaron dos grupos de acuerdo a su calificación en la Escala de ansiedad social para adolescentes: el primero con bajo $(n=2640)(\bar{x}=25.9, D E=5.8)$ y el segundo con alto grado $(\mathrm{n}=524)(\bar{x}=44.2, \mathrm{DE}=5.7)$ de ansiedad social, y se compararon sus niveles de consumo de alcohol, encontrando que: contrario a lo que establece la literatura del tema en adultos, el patrón de consumo de alcohol en los grupos de adolescentes de alta y baja ansiedad social, no presentó diferencias estadísticamente significativas $\left(\mathrm{X}^{2}=1.201, \mathrm{gl}=2, p=.361\right)$. Probablemente, las diferencias reportadas en el consumo de alcohol entre adultos social y no socialmente ansiosos, surjan sólo en ciertas variantes de la fobia social, o bien, se requiere de otras variables mediadoras, entre fobia social y el consumo abusivo de alcohol. Palabras clave: Fobia social, ansiedad social, patrón de consumo de alcohol, adolescentes. 
Abstract

The study of social phobia is gradually increasing, arguing association between social phobia and multiple measures of dysfunction. After institutional approval, and in accordance with the ethical principles required for social research, socio-demographic data, measurements of social anxiety and alcohol consumption pattern, were obtained from 3164 Mexican adolescents volunteers (men and women) ages 12-18 years $(\bar{x}$ $=14.7, S D$ 1.7), in different school schedule (morning or afternoon) and with record of their extracurricular activities" (to participate or not in sports, arts and been involved or not in a dating relationship). The participants were divided into two groups, according to the score on the Social Anxiety Scale for Adolescents: high $(\mathrm{n}=524)(\bar{x}=44.2, S D=5.7)$, and low $(\mathrm{n}=2640)(\bar{x}=25.9, S D=5.8)$, degree of social anxiety. Also, their levels of alcohol consumption were compared. The results of the study showed that: In contrast to the suggested by the literature in adults, the pattern of alcohol consumption in adolescents of low social anxiety group and the high social anxiety group, did not presented statistically significant differences $\left(X^{2}=1.201\right.$, $\mathrm{gl}=2, \mathrm{p}=.361)$. A likely explanation of these reported differences in drinking among socially anxious and not socially anxious adults, is that they occur only in certain variants of social phobia, or maybe, it requires other mediating variables between social phobia and alcohol abuse.

Key words: Social phobia, social anxiety, alcohol consumption pattern, teens.

Introducción

Es frecuente que algunas personas experimenten cierto malestar ante situaciones como hablar con un desconocido, frente a un grupo, o incluso con algunos conocidos o amigos; tal emoción se conoce como ansiedad social y se presenta en la población general, en un continuo que va de leve a grave (Book \& Randall, 2002).

El diagnóstico clínico de fobia social (en adelante FS) también Ilamada Trastorno de Ansiedad Social, se asigna cuando el grado de ansiedad social, representa un miedo persistente y acusado ante situaciones de interacción social, en las cuales, la persona cree que podría ser evaluada negativamente o humillada (American Psychiatric Association [APA], 2000) y ante tales circunstancias, evita deliberadamente situaciones sociales.

La FS dificulta al individuo el establecimiento y mantenimiento de las relaciones interpersonales (Alden \& Taylor, 2004), mismas que constituyen un aspecto cardinal del comportamiento humano, en particular entre los adolescentes ya que su independencia gradual de los adultos, intensifica como contraparte sus relaciones con los pares (Wissink, Dekovic, \& Meijer, 2009; Zubeidat, Sierra, \& Fernández, 2006), además, como señalan La Greca y López (1998), las amistades adolescentes pueden tener una naturaleza protectora o ser un factor de riesgo para algunos trastornos externalizados.
En virtud de lo anterior, el propósito del presente trabajo fue estimar las posibles diferencias en el patrón de consumo de alcohol, en una muestra de adolescentes escolarizados (hombres y mujeres) entre 12 y 18 años de edad, de distintos grados de ansiedad social (alto o bajo), turno escolar (matutino o vespertino) y actividades extracurriculares (realizar o no actividades deportivas, artísticas y tener o no una relación de noviazgo). Sin establecer atribuciones de causalidad.

Desde el inicio del SXXI, se intensificó el interés por estudiar a la fobia social, las primeras investigaciones se realizaron en muestras de adultos (Kashdan \& Herbert, 2001), sin embargo, frente a una creciente cantidad de investigaciones que reportan correlaciones positivas entre la ansiedad social y diversas problemáticas adolescentes (Kashdan, Collins, \& Elhai, 2006), se le considera como un factor de riesgo que puede impactar negativamente el proceso de adaptación en ese sector de la población (Rao et al., 2007).

Los modelos cognitivos de la fobia social (Clark \& Wells, 1995; Rapee \& Heimberg, 1997; Turk, Lerner, Heimberg, \& Rapee, 2001) coinciden en que los fóbicos sociales, interpretan las situaciones sociales ambiguas, como negativas o amenazantes, tienden a desestimar su trabajo y atributos personales en situaciones sociales, evidencian 
autoevaluaciones negativas y menor sensibilidad a la información positiva (Kashdan \& Savostyanova, 2011), también temen comportarse en tal forma que otras personas les consideren débiles o torpes, y que se den cuenta de su ansiedad, es decir que: la preocupación fundamental de los fóbicos sociales, es el miedo a ser evaluado negativamente (Miers, Blöte, Bokhorst, \& Westenberg, 2009; Schultz \& Heimberg, 2008).

En México, la Encuesta de Salud Mental de Adolescentes de la Ciudad de México (Benjet et al., 2009), ubica a la fobia social en el segundo lugar entre los diagnósticos de mayor frecuencia en la adolescencia, después de las fobias específicas, con prevalencia de $10 \%$ para el sexo masculino y $12.5 \%$ para el femenino, sin embargo, el diagnóstico de fobia social, se asocia con discapacidad menor y por otra parte, como señalan Benjet et al. (2009), los adolescentes con trastornos de ansiedad, son los que menos buscan atención por ese motivo, en los servicios de salud.

Respecto del consumo de alcohol, la proporción de personas que cumple los criterios de abuso/dependencia de alcohol en México, es mayor a cuatro millones (Encuesta Nacional de Adicciones [ENA] 2008), la Encuesta de Consumo de Drogas en Estudiantes 2009 de la Ciudad de México (Villatoro et al., 2011) reitera la edad de inicio en el consumo de alcohol a los 12.6 años y aunque existe una heterogeneidad en las trayectorias del consumo (Chassin, Pitts, \& Prost, 2002), "el abuso del alcohol es un factor de riesgo para la mortalidad prematura o días vividos sin salud" (del bosque et al., 2012, p.165).

\section{Aproximaciones teóricas de la relación ansiedad-consumo de alcohol}

Si bien desde la década de los años 50, se establecen relaciones entre los trastornos de ansiedad en general y los trastornos por consumo de alcohol y otras sustancias, los mecanismos causales que subyacen a dicha relación no son suficientemente claros. Algunas propuestas teóricas para explicar dicha asociación son las siguientes:

1) Modelo Causal: su premisa es que la presencia de uno de los trastornos conduce directamente al desarrollo del otro. A este grupo pertenecen la Teoría de la Reducción de la Tensión (Conger, 1956), según la cual, la tensión, la ansiedad o el estrés juegan un papel instigador en el consumo del alcohol, y a su vez, constituye una respuesta instrumental de escape reforzada por sus propiedades de reducción de la tensión (Higgins \& Marlatt, 1975), en este mismo renglón se ubica la Teoría de la Automedicación (Khantzian, 1987; Thomas, Randall, \& Carrigan, 2003), versión reformulada de la Teoría de la Reducción de la Tensión (Conger, 1956). La teoría de la automedicación establece que las personas con un trastorno de ansiedad primario, tienden a desarrollar trastornos por uso de sustancias como resultado del intento de autorregular sus síntomas de ansiedad (Robinson, Sareen, Cox, \& Bolton, 2009).

2) Modelo Causal Indirecto: plantea que la presencia de uno de los trastornos, incrementa el riesgo individual de desarrollar el otro, como es el caso de la Teoría de la Respuesta al Estrés (Sher, \& Levenson, 1982).

3) Modelo de Relación no Causal: propone que factores comunes pueden sumarse para aumentar el riesgo de desarrollar ambos trastornos. En los tres casos, persiste la hipótesis global de que el alcohol actúa como reforzador negativo para reducir el estrés o el afecto negativo.

Algunos estudios epidemiológicos afirman que existen diferentes relaciones entre cada uno de los trastornos de ansiedad y los trastornos por consumo de alcohol, y que entre ellos, la agorafobia y la fobia social, son propensas a la automedicación (Kushner, Sher, \& Beitman 1990).

Fobia social y consumo de alcohol La propuesta en torno a la relación entre fobia social y el abuso o dependencia del alcohol (Zimmermann et al., 2003), es controversial, el debate plantea falta de claridad en la relación entre fobia social y la cantidad-frecuencia del consumo, en los mecanismos subyacentes a la posible relación entre ambos problemas, así como en la direccionalidad de la misma (Anderson, Grunwald, Bekman, Brown, \& Grant, 2011).

Los estudios que apoyan la relación fobia socialconsumo de alcohol, sugieren que quienes presentan ansiedad social clínicamente significativa e incluso ansiedad social subclínica, presentan mayor incidencia de problemas relacionados con el consumo de alcohol, que la población general (e. g., Buckner, Tímpano, Zvolensky, Sachs-Ericsson 
y Schmidt, 2008; Buckner, Eggleston, \& Schmidt, 2006; Crum \& Pratt, 2001), consideran que el papel de la fobia social como factor de riesgo asociado al consumo excesivo de alcohol, es un campo emergente para la investigación, puesto que el abuso y dependencia del alcohol durante la adolescencia, siguen siendo una prioridad en Salud Pública, merced a sus reconocidas consecuencias adversas -por ello- es fundamental la identificación de factores de riesgo modificables (Pons \& Buelga, 2011; Stice, 2002), como lo establece el enfoque preventivo de la psicología de la salud (Ezpeleta, 2005; Flores, 2007).

En este contexto, Schneier et al. (2010), con base en los resultados de una encuesta epidemiológica nacional realizada en una muestra representativa de los Estados Unidos, reportaron que la prevalencia de la comorbilidad entre los trastornos por consumo de alcohol, y la fobia social a lo largo de la vida, se presentó en un $2 \%$ de la población general, concluyeron que dicho diagnóstico dual es frecuente y que ha permanecido largamente sin atención. Por su parte, Wancata, Fridl y Friedrich (2009) reportaron una asociación (Razón de Momios=RM) durante doce meses en la población general entre fobia social y abuso del alcohol $(R M=2.7)$, fobia social y dependencia del alcohol $(R M=2.7)$ en países europeos. En el mismo sentido, Thomas, Randall, Book y Randall (2008), documentaron la relación entre fobia social y uso de alcohol, a partir de estudios en población comunitaria, atención primaria y en población clínica que recibía tratamiento para el abuso de sustancias; que la FS coexiste con trastornos por consumo de sustancias, incluido el alcohol en adultos, y que sin tratamiento, la FS puede asociarse con el desarrollo, mantenimiento y recaída en trastornos por consumo de alcohol. No obstante, los mismos autores plantean que los estudios en población adolescente, aún son insuficientes para comprender la relación entre fobia social y abuso del alcohol.

Botella, Baños y Perpiñá (2003) refieren que la proporción de personas que consumen alcohol entre los fóbicos sociales, es mayor que la observada en otros trastornos de ansiedad. Book y Randall (2002) estiman que una de cada cinco personas con diagnóstico de FS, presenta algún trastorno por consumo de alcohol (abuso o dependencia).
Al respecto, Zimmermann, et al. (2003), concluyeron con base en datos de una encuesta comunitaria aplicada a adultos, que el trastorno de pánico y la fobia social, son predictores de problemas subsecuentes con el alcohol, por lo que proponen mayor investigación, para comprender el valor potencial del tratamiento temprano de estos trastornos de ansiedad, como estrategia para prevenir trastornos secundarios por consumo de alcohol. En este mismo sentido, Arehart-Treichel (2007) y Kessler (2003) consideran que es posible incidir sobre problemas de salud pública como el consumo del tabaco y dependencia del alcohol, interviniendo a través de programas preventivos sobre la ansiedad social.

En sentido opuesto, Ham y Hope (2005), consideran que la ansiedad social incluso puede calificarse como un factor protector para el consumo abusivo o dependiente del alcohol u otras sustancias. Coincidentemente, Eggleston, Woolaway-Bickel, y Schmidt (2004), reportaron una correlación inversamente relacionada entre la ansiedad social y la frecuencia del consumo de alcohol.

El tema se ha investigado muy poco en México, como se hizo evidente en la búsqueda de información nacional realizada en diversas bases de datos, utilizando los descriptores, ansiedad social, fobia social, y abuso o dependencia de alcohol, sin identificar investigaciones mexicanas, que hayan estudiado la relación entre ambos problemas entre 2005 y 2011.

Considerando lo anterior, el presente trabajo se planteó como pregunta de investigación: cuales son las diferencias en el patrón de consumo de alcohol, en una muestra de adolescentes escolarizados (hombres y mujeres) entre 12 y 18 años de edad, de distintos grados de ansiedad social (alto o bajo), turno escolar (matutino o vespertino) y actividades extracurriculares (realizar o no actividades deportivas, artísticas y tener o no una relación de noviazgo) planteando como pregunta de investigación.

\section{Método}

\section{Participantes}

Se realizó un estudio de campo, no experimental, transversal correlacional, con un muestreo no probabilístico y accidental de participantes 
voluntarios (Kerlinger \& Lee, 2002).

Se evaluó a 3244 participantes, de los cuales se eliminó a 80 que respondieron en forma incompleta o inadecuada los instrumentos, por lo tanto, la muestra se integró con 3164 adolescentes escolarizados de los cuales, 1738 (54.9\%) fueron de sexo femenino y 1426 de sexo masculino (45.1\%), provenientes de escuelas oficiales de nivel medio y medio superior de la Ciudad de México, entre 12 y 18 años de edad ( $\bar{x}=14.7, D E 1.7)$.

Como indica la tabla 1 , los datos descriptivos de los participantes en términos de sus características sociodemográficas, señalan un mayor número de participantes del sexo femenino, del turno matutino, y de quienes reportaron mayoritariamente, sí realizar actividades deportivas. Son mayoría quienes refirieron no practicar actividades artísticas y minoría, quienes reportaron tener una relación de noviazgo.

\section{Instrumentos}

1. Cuestionario de Datos Generales: Se solicitaron datos de edad, sexo, escuela, grado escolar, turno escolar, práctica o no de algún deporte de forma regular, práctica o no de alguna actividad artística de forma regular y tener o no una relación de noviazgo.

2. El grado de ansiedad social (bajo o alto) se estimó a partir de la puntuación obtenida en la Escala de Ansiedad Social para Adolescentes (SAS-A) (La Greca \& López, 1998). Que es un instrumento de auto-reporte acerca de la ansiedad social. La SAS-A contiene tres subescalas que son: a) Miedo

Tabla 1.

Distribución de la muestra estudiada por sexo, nivel educativo, turno escolar y actividades extracurriculares

\begin{tabular}{|c|c|c|c|c|c|c|c|}
\hline \multirow[t]{2}{*}{ Características de la Muestra } & & \multicolumn{2}{|c|}{ Hombres } & \multicolumn{2}{|c|}{ Mujeres } & \multicolumn{2}{|c|}{ Totales } \\
\hline & & $\mathrm{n}$ & $\%$ & $\mathrm{n}$ & $\%$ & $\mathrm{n}$ & $\%$ \\
\hline \multicolumn{8}{|l|}{ Escolaridad } \\
\hline Secundaria & & 667 & 21.1 & 687 & 21.7 & 1354 & 42.8 \\
\hline \multirow[t]{2}{*}{ Bachillerato } & & 759 & 24.0 & 1051 & 33.2 & 1810 & 57.2 \\
\hline & totales & 1426 & 45.1 & 1738 & 54.9 & 3164 & 100 \\
\hline \multicolumn{8}{|l|}{ Turno Escolar } \\
\hline Matutino & & 981 & 31.0 & 1314 & 41.5 & 2295 & 72.5 \\
\hline \multirow[t]{2}{*}{ Vespertino } & & 445 & 14.1 & 0424 & 13.5 & 869 & 27.5 \\
\hline & totales & 1426 & 45.1 & 1738 & 54.9 & 3164 & 100 \\
\hline \multicolumn{8}{|l|}{ Actividades extracurriculares } \\
\hline Realizan actividad deportiva & & 1044 & 33.0 & 696 & 22.0 & 1740 & 55.0 \\
\hline \multirow[t]{2}{*}{ NO Realizan actividad deportiva } & & 0380 & 12.0 & 1042 & 32.9 & 1422 & 44.9 \\
\hline & totales & 1424 & 45.0 & 1738 & 54.9 & 3162 & 99.9 \\
\hline Practican actividades artísticas & & 501 & 15.8 & 0637 & 20.1 & 1138 & 35.9 \\
\hline \multirow[t]{2}{*}{ NO Practican actividades artísticas } & & 925 & 29.2 & 1101 & 34.8 & 2026 & 64.0 \\
\hline & totales & 1426 & 45.1 & 1738 & 54.9 & 3164 & 99.9 \\
\hline Tienen relación de noviazgo & & 515 & 16.3 & 0609 & 19.2 & 1124 & 35.5 \\
\hline \multirow[t]{2}{*}{ NO Tienen relación de noviazgo } & & 911 & 28.8 & 1129 & 35.7 & 2040 & 64.5 \\
\hline & totales & 1426 & 45.1 & 1738 & 54.9 & 3164 & 100 \\
\hline
\end{tabular}


a la Evaluación Negativa (FNE por sus siglas en inglés), b) Evitación Social y Estrés Específico a Situaciones Sociales Nuevas (SAD-New por sus siglas en inglés) y c) Evitación y estrés ante situaciones sociales en general (SAD-G). Respecto de la validez de constructo, Inglés et al. (2010) refieren que se ha ratificado la estructura factorial de tres dimensiones propuesta en el estudio original (La Greca \& López, 1998), en diversas muestras de adolescentes.

En el presente trabajo se aplicó la versión Española de la SAS-A (Olivares, Ruiz et al., 2005), que se validó previamente en una muestra de adolescentes mexicanos, obteniendo una confiabilidad de $(\alpha=.85)$. Se consideró alto grado de ansiedad social si la puntuación obtenida fue igual o mayor a una desviación estándar por encima de la puntuación media $(\bar{x}=44.2, S D=5.7)$. El resto de los participantes se incluyó en la categoría: bajo grado de ansiedad social $(\bar{x}=25.9, S D=5.8)$.

3. El consumo riesgoso de alcohol fue medido a través de la Escala AUDIT (Alcohol Use Disorders Identification Test) (Saunders, Aasland, Babor, De la Fuente, \& Grant, 1993). Se trata de un instrumento auspiciado por la Organización Mundial de la Salud (OMS) para evaluar la magnitud del consumo de alcohol. A partir de este instrumento, la OMS estableció los conceptos de trastornos por consumo de alcohol; consumo de riesgo y consumo dañino o perjudicial, incorporándolos al ya existente de dependencia del alcohol (Guzmán, Pedrão, Rodríguez, López, \& Esparza, 2007).

El AUDIT está compuesto por 10 reactivos en formato de escala Likert con 5 opciones de respuesta. Las 3 primeras preguntas miden la frecuencia y cantidad del consumo, mientras que las preguntas 4 a 6, evalúan síntomas de dependencia de alcohol y de la 7 en adelante, hacen referencia a los trastornos causados por el alcohol (Lucio, Gómez, Morales, \& Pérez, 2009), provee información acerca del consumo en el año anterior, el mes anterior, semanalmente y diariamente. Los sujetos pueden clasificarse en tres categorías: a) consumo no perjudicial de alcohol (abstinencia o bajo consumo), b) consumo riesgoso o problemático y c) dependencia del alcohol (Fantín \& Barbenza, 2007).

En México, Lucio et al. (2009) reportaron una estructura bifactorial: el primer factor identifica trastornos por consumo de alcohol con $(\alpha$ $=.73$ ) y explica el $41.6 \%$ de la varianza total. El segundo factor establece la frecuencia y cantidad del consumo de alcohol, con $(\alpha=.85)$ y 11.4 $\%$ de varianza explicada. El coeficiente Alfa de Cronbach de la escala total es de .82, mostrando con ello una consistencia interna de media a robusta. Lucio et al. (2009) concluyeron que las propiedades psicométricas de la escala AUDIT son adecuadas para ser utilizada en adolescentes escolarizados. Para los adolescentes se considera que una puntuación de 0 a 2, indica un consumo sin riesgo y de 3 puntos en adelante, se configura el consumo riesgoso y dañino de alcohol (Díaz et al., 2009).

\section{Procedimiento}

Habiendo gestionado la autorización institucional y asumiendo los principios éticos de autonomía, beneficencia, privacidad y protección a los derechos humanos, se invitó a los participantes a colaborar voluntariamente en el estudio -para ello- se les informó acerca de los objetivos y procedimiento del mismo, así como las condiciones de anonimato y confidencialidad de los datos proporcionados. Una vez obtenido el asentimiento de los adolescentes, se procedió a la aplicación de los instrumentos, en sus respectivas aulas, siguiendo todos los aplicadores (estudiantes de los últimos semestres de la licenciatura en psicología, o psicólogos recién graduados) la misma secuencia.

Para el análisis de datos, se utilizó el paquete estadístico para las ciencias sociales (SPSS) versión 15 para Windows.

\section{Resultados}

\section{Distribución de la ansiedad social y el consumo de alcohol}

En esta muestra, la media de la puntuación general de la ansiedad social $(\bar{x}=29.02, D E=8.9)$, no señaló diferencias estadísticamente significativas $(t=3086$, $1.30, p>.05)$ entre hombres $(\bar{x}=28.79 D E=8.7)$ y mujeres $(\bar{x}=29.20, D E=9.1)$. Los participantes con alto nivel de ansiedad social, fueron 524 (16.5\%) $(\bar{x}=44.2, D E=5.7)$, con una mayor frecuencia entre mujeres $(9.6 \%)$ que entre hombres $(6.9 \%)$, el resto de los participantes (2640) (83.4\%) obtuvieron 
bajo nivel de ansiedad social $(\bar{x}=25.9, D E=5.8)$.

En cuanto al consumo de alcohol, la media general fue de $(\bar{x}=2.96, D E=4.4)$, con diferencias estadísticamente significativas $(t=3162,-2,2.59, p \leq$ $.01)$ entre hombres $(\bar{x}=3.18, D E=4.5)$ y mujeres $(\bar{x}=2.7, D E=4.3)$. El $65.3 \%$ de los participantes refirió un consumo sin riesgo, mientras que el $34.7 \%$ de los adolescentes presentó un consumo riesgoso o problemático $(\bar{X}=7.69, D E=4.66)$, de ellos $17.1 \%$ fueron hombres y $17.5 \%$ mujeres.

Con la finalidad de estimar las diferencias en las puntuaciones medias, obtenidas en ansiedad social y en el consumo de alcohol, en función de las variables sexo, turno escolar y actividades extracurriculares, se realizaron pruebas $t$ para muestras independientes. Los resultados, como se muestra en la tabla 2, mostraron que la ansiedad social no registró diferencias estadísticamente significativas por sexo, se presentó con mayor frecuencia en el turno matutino, entre quienes reportaron no practicar actividades deportivas y entre los que dijeron no tener una relación de noviazgo.

El consumo de alcohol, fue mayor entre los hombres, en el turno vespertino, y entre quienes reportaron sí realizar actividades deportivas y entre quienes afirmaron sí tener una relación de noviazgo.

Considerando el bajo o alto grado de ansiedad social y la edad de los adolescentes, se realizó un

Tabla 2.

Diferencia de medias en ansiedad social y consumo de alcohol en función de las variables sociodemográficas.

\begin{tabular}{|c|c|c|c|c|c|c|}
\hline \multirow{2}{*}{$\begin{array}{l}\text { Características } \\
\text { sociodemográficas }\end{array}$} & \multicolumn{3}{|c|}{ Ansiedad social } & \multicolumn{3}{|c|}{ Consumo de alcohol } \\
\hline & $\bar{x}$ & $D E$ & $t$ & $\bar{x}$ & $D E$ & $t$ \\
\hline \multicolumn{7}{|l|}{ Sexo } \\
\hline Mujeres & 29.20 & 9.11 & $3086,1.30$ & 2.78 & 4.32 & $3162,-2.59 * *$ \\
\hline Hombres & 28.79 & 8.74 & & 3.19 & 4.57 & \\
\hline \multicolumn{7}{|l|}{ Turno Escolar } \\
\hline Matutino & 29.35 & 9.21 & $3162,3.45^{* * *}$ & 2.76 & 4.38 & $3162,-4.16^{* * *}$ \\
\hline Vespertino & 28.12 & 8.14 & & 3.90 & 4.56 & \\
\hline \multicolumn{7}{|c|}{ Actividades Extracurriculares } \\
\hline Si deporte ${ }^{1}$ & 28.55 & 8.93 & $3160,3.18^{* * *}$ & 3.14 & 4.60 & $3160,2.38^{*}$ \\
\hline No deporte ${ }^{2}$ & 29.57 & 8.94 & & 2.76 & 4.22 & \\
\hline Si artísticas ${ }^{3}$ & 28.84 & 9.01 & $3162,0.83$ NS & 2.93 & 4.29 & $2463,0.30$ \\
\hline No artisticas ${ }^{4}$ & 29.12 & 8.91 & & 2.98 & 4.53 & \\
\hline Si Novio ${ }^{5}$ & 28.18 & 9.11 & $3162,3.90^{* * *}$ & 3.83 & 4.85 & $3162,8.25^{* *}$ \\
\hline No Novio ${ }^{6}$ & 29.48 & 8.89 & & 2.48 & 4.12 & \\
\hline
\end{tabular}

1 Sí reporta actividades deportivas; 2 No reporta actividades deportivas; 3 Sí reporta actividades artísticas; 4 No reporta actividades artísticas; 5 Sí reporta relación de noviazgo; 6 No reporta relación de noviazgo. NS = diferencia no significativa. 
Tabla 3.

Diferencias por edad entre adolescentes con alto y bajo grado de Ansiedad Social.

\begin{tabular}{ccccccc}
\hline & & \multicolumn{5}{c}{ Puntuaciones medias en consumo de alcohol } \\
\cline { 3 - 6 } Edad & $\%$ & Alta ansiedad social & Baja ansiedad social & Diferencias \\
& & $\overline{\mathrm{x}}$ & $D E$ & $\overline{\mathrm{x}}$ & $D E$ & entre $\overline{\mathrm{x}}$ \\
\hline 12 & 14.8 & 1.74 & 3.52 & 1,34 & 2.97 & \\
13 & 12.6 & 2.00 & 3.82 & 2.10 & 3.69 & \\
14 & 17.1 & 4.21 & 5.86 & 3.54 & 5.18 & \\
15 & 28.3 & 2.68 & 3.59 & 3.18 & 4.37 & \\
16 & 7.6 & 3.65 & 4.99 & 3.61 & 4.52 & \\
17 & 16.9 & 3.11 & 4.45 & 3.44 & 4.50 & \\
18 & 2.3 & 8.67 & 8.33 & 4.72 & 5.29 & $3.94^{*}$ \\
\hline
\end{tabular}

$* \mathrm{p} \leq .05$

Tabla 4.

Análisis de regresión logística de ansiedad social sobre las variables sociodemográficas

\begin{tabular}{lccc}
\hline Variables predictoras estadísticamente significativas & $\beta$ & RM & IC 95\% \\
\hline Hacer deporte & $-.180^{*}$ & 0.836 & $0.710-0.984$ \\
Hacer actividades artísticas & -.071 & 0.931 & $0.785-1.105$ \\
Tener novio & -.122 & 0.885 & $0.745-1.051$ \\
\hline
\end{tabular}

Regresión logística método adelante Wald.

$\mathrm{RM}=$ razón de momios (odds ratio).

IC $95 \%=95 \%$ intervalo de confianza.

análisis de varianza simple por edad cuyo análisis de las medias marginales (ver tabla 3), mostró que el consumo de alcohol, únicamente en los participantes de 18 años edad, registró diferencias estadísticamente significativas, siendo mayor el consumo de los participantes de 18 años de edad, con alto grado de ansiedad social.

El siguiente análisis fue una regresión logística estableciendo como variable predictora la ansiedad social y como variable criterio, el ser o no abusador del alcohol. Los resultados indicaron que aunque el estadístico de Wald fue significativo (3.82 $p=.050)$, el impacto de la ansiedad sobre el consumo de alcohol no es fuerte ya que la razón de momios fue ligeramente menor a 1 (.992).

Asimismo, para determinar la relación de la ansiedad social con las variables sociodemográficas, se hicieron análisis de regresión logística considerando como variables criterio a la actividad deportiva, artística y la de tener relaciones de pareja. Los resultados señalan que entre las variables criterio incluidas, únicamente hacer deporte se asocia con la ansiedad social, la población con mayor grado de ansiedad social tiene menor probabilidad de involucrarse en la práctica de deportes.

Finalmente, no se identificaron diferencias estadísticamente significativas en el patrón de 
consumo de alcohol $\left(X^{2}=1.201, g l=2, p=.361\right)$ entre los participantes de alta y los de baja ansiedad social.

\section{Conclusiones}

La ansiedad social mostró predominio femenino en el grupo de alta ansiedad social, sin registrar diferencias estadísticamente significativas entre hombres y mujeres. Esto es consistente con los resultados de la Encuesta Mexicana de Salud Mental Adolescente (Benjet et al., 2009), con los estudios clásicos de Kessler et al. (1994) y, los de Stoyanova y Hope (2012), lo cual reitera una mayor vulnerabilidad en las mujeres para desarrollar una gama de miedos a lo largo de la vida, y mayor probabilidad que la de los hombres, a presentar algún trastorno de ansiedad (García-López, Piqueras, Díaz-Castela, \& Inglés, 2008; Stoyanova \& Hope, 20012; Xu et al., 2012). Los participantes con alto grado de ansiedad social, reportaron menor involucramiento en actividades deportivas y en relaciones románticas.

En cuanto a las diferencias por actividades extracurriculares, se observó mayor grado de ansiedad social entre los adolescentes que reportaron no realizar actividades deportivas y entre los que afirmaron no tener una relación de noviazgo, lo cual era de esperarse si se considera que la interpretación errónea de los estímulos sociales, conduce a los ansiosos sociales a evaluarlos como peligrosos y por lo tanto, el miedo y la evitación, se manifiestan como un menor involucramiento en diligencias que demandan interacción directa con otros y ser observados durante el desarrollo de actividades como las deportivas, o de otro tipo (Rao et al.,2008). En este sentido, Borda (2009) señala que la intensidad de la actividad física adolescente se relaciona con la autoeficacia, misma que se encuentra disminuida en los adolescentes ansiosos sociales (Kashdan \& Roberts, 2006).

En el rubro del consumo de alcohol, éste fue mayor entre los hombres, en el turno vespertino y en quienes refirieron Ilevar a cabo prácticas deportivas e involucramiento en relaciones de noviazgo.

Se revalidó que a mayor edad, mayor consumo, y que ser hombre incrementa el riesgo para el consumo problemático de alcohol y otras sus- tancias consistentemente con lo reportado en estudios mexicanos (Pérez \& Lucio-Gómez, 2010; Díaz et al., 2009; Villatoro, et al., 2009). Asimismo, se confirmó que la asistencia al turno vespertino es un factor de riesgo para el consumo de alcohol como lo han advertido algunos investigadores (Nuño-Gutiérrez, Álvarez-Nemegyei, Madrigal-de León, \& Rasmussen, 2005; Pérez \& Lucio-Gómez, 2010), que afirman que en dicho turno, se concentran los estudiantes de mayor edad, así como los de menores calificaciones; o, los que presentan situaciones académicas irregulares.

En el caso específico del abuso del alcohol, no hubo diferencias estadísticamente significativas por sexo $(t=1.29, p=.897)$, lo cual concuerda con los datos reportados en las Encuestas Nacionales de Adicciones, 2002, 2008 y 2011. En ellas, se reitera una tendencia al incremento del abuso en las adolescentes mujeres.

$\mathrm{Al}$ respecto de las diferencias en consumo de alcohol, por actividades extracurriculares, fue notorio un mayor consumo de alcohol entre los adolescentes que reportaron sí realizar práctica deportiva, y en quienes informaron sí tener una relación de noviazgo. En este tenor, los contextos socio-ambientales facilitan el consumo de alcohol y el ejercicio vigoroso en asociación, lo cual no es de sorprender, si se analizan las estrategias de marketing que son dirigidas a esta población, a pesar de la regulación, y de los señalamientos que hacen los expertos en contra de ellas (MedinaMora et al., 2010).

Finalmente, el consumo riesgoso o problemático de alcohol, se comportó de la misma forma entre los adolescentes con alto grado y los de bajo grado de ansiedad social, por lo que se puede afirmar que: durante la adolescencia, la relación entre ansiedad social y consumo de alcohol, es diferente de la que se ha descrito en otros estudios entre adultos.

\section{Discusión}

Características normativas de la adolescencia como los cambios neuroendócrinos asociados al desarrollo, el logro de las operaciones formales, egocentrismo e incremento de los miedos sociales (Ellis et al., 2012), favorecen la participación de los adolescentes en conductas de riesgo (com- 
portamientos que involucran la integridad física y psicológica de los adolescentes, activados por experiencias vitales estresantes, coincidentes con vulnerabilidades personales o biológicas [Campo-Arias, Cogollo, \& Díaz, 2008]), el planteamiento de que la ansiedad social clínicamente significativa, o aún en el nivel subclínico, podría constituir una de estas vulnerabilidades que se constituyen en factor de riesgo para diferentes comportamientos disfuncionales como el consumo problemático de alcohol (Buckner \& Turner, 2009), de ser comprobado, permitiría que, además de los ya conocidos factores de riesgo que inciden sobre el consumo excesivo de alcohol, se identificara un nuevo elemento sobre el cual investigar, conocer su papel como factor de riesgo (por ejemplo, etiológico, de mantenimiento, mediador), el tamaño del efecto al incidir sobre él etc. Sin embargo, en el presente estudio, las diferencias en el patrón de consumo de alcohol entre los adolescentes con alto y los de bajo nivel de ansiedad social, no fueron estadísticamente significativas $\left(X^{2}=1.201, g l=2, p=.361\right)$. Estos resultados apoyan al grupo de investigadores que como Buckner, Ecker, y Proctor (2011), sugieren que las diferencias al respecto señaladas en otros estudios, podrían ser simplemente consecuencias de evaluar diferentes aspectos de la ansiedad social y del consumo de alcohol -por ejemplo- la edad de inicio, cantidad y frecuencia del consumo, búsqueda de tratamiento, recaídas, abuso o dependencia. Es importante destacar es que en el presente estudio, las diferencias estadísticamente significativas entre ansiosos y no ansiosos sociales únicamente se identificaron en la población de 18 años, ello llevaría a preguntar si es la variable edad, un factor relevante para identificar dicha asociación y da una pauta para continuar estudiando el tema, con diferentes grupos de edad.

A su vez, Buckner, Schmidt, et al. (2008), afirman que la fobia social, es un predictor específicamente para la dependencia, pero no para el abuso del alcohol. Apoyan la hipótesis de que las personas con alto grado de ansiedad social son más vulnerables a los efectos ansiolíticos del alcohol, pero recomiendan mayor investigación, para precisar si otras variables (p. ej. nivel de estrés, apoyo social), median la relación entre el consumo abusivo o dependencia del alcohol y un elevado nivel de ansiedad social.

Por otra parte, es importante considerar que aún existen ciertas inconsistencias en la caracterización de la ansiedad social, por ejemplo, Kashdan y McKnight (2010) apuntan la existencia de cuando menos dos subgrupos de ansiosos sociales, independientemente de los señalados en el DSM-IV TR. El subgrupo inhibido es el prototípico, se caracteriza por un patrón de inhibición conductual, aversión al riesgo y tendencia a la evitación-escape. El subgrupo desinhibido, presenta conductas egoístas e impulsivas en una intensa búsqueda de novedades y emociones. Este subtipo no ha sido considerado en la mayoría de las descripciones de la ansiedad social, de tal suerte que se podrían estar extendiendo erróneamente las características del grupo inhibido al desinhibido.

Si la FS incluye perfiles heterogéneos (Kashdan \& McKnight, 2010), no todos los individuos que presentan FS, cumplen con el estereotipo del grupo prototípico, por lo tanto, las diferencias en las estrategias de procesamiento cognitivo y de regulación emocional, entre los distintos grupos de fóbicos sociales, podrían conducir a diferentes resultados. De acuerdo con esta clasificación, exclusivamente el grupo desinhibido podría presentar riesgo de consumo excesivo de alcohol y en el presente estudio, no fueron considerados los subtipos de ansiosos sociales.

Finalmente, es importante considerar las limitaciones del presente estudio, entre ellas, su diseño, el muestreo intencional y los instrumentos de tamizaje utilizados. Se requiere de estudios longitudinales, diagnóstico clínico para analizar con mayor certeza, la posible asociación en el tiempo, entre la fobia social y los trastornos por consumo de alcohol en adolescentes de diferentes tipos, pues como señalan Ham y Hope (2005), los estudios empíricos sobre el tema son insuficientes, y sus resultados no concluyentes.

\section{Referencias}

Alden, L., \& Taylor, C. (2004). Interpersonal processes in social phobia. Clinical Psychology Review, 24(7), 857-882. doi:10.1016/j. cpr.2004.07.006

American Psychiatric Association (2000). Di- 
agnostic and Statistical Manual of Mental Disorders, DSM-IV. New York. Washington DC. American Psychiatric Association.

Anderson, K., Grunwald, I., Bekman, N., Brown, S., \& Grant, A. (2011). To drink or not to drink: Motives for use and nonuse in adolescence. Addictive Behaviors, 36(10), 972979. doi:10.1016/j.addbeh.2011.05.009

Arehart-Treichel, J. (2007). Social anxiety in teenagers raises substance-Abuse Risk. Psychiatric News, 42(11), 15-15.

Bacon, A., \& Ham, L. (2010). Attention to social threat as a vulnerability to the development of comorbid social anxiety disorder and alcohol use disorders: An Avoidance-Coping Cognitive Model. Addictive Behaviors, 35(11), 925-939. doi:10.1016/j.addbeh.2010.06.002

Benjet, C., Borges, G., Medina-Mora, M., Méndez, E., Fleiz, C., Rojas, E., \& Cruz, C. (2009). Diferencias de sexo en la prevalencia $y$ severidad de trastornos psiquiátricos en adolescentes de la Ciudad de México. Salud Mental, 32(2), 155-163.

Book, S., \& Randall, C. (2002). Social Anxiety disorder and Alcohol Use. Alcohol Research \& Health, 26(2), 130-135.

Borda, R. (2009). Factores psicosociales asociados a la práctica de la Actividad Física en Adolescentes de la Localidad de Engativa, de Acuerdo con la Teoría de la Motivación Protectora. Típica, 5(2), 145-156.

Botella, C., Baños, R., \& Perpiñá, C. (2003). Fobia social: Avances en la psicopatología, la evaluación y el tratamiento psicológico del trastorno de ansiedad social. Barcelona, Paidós.

Buckner, J., Ecker, A., \& Proctor, S. (2011). Social anxiety and alcohol problems: The roles of perceived descriptive and injunctive peer norms. Journal of Anxiety Disorders, 25(5), 631-638. doi:10.1016/j.janxdis.2011.02.003

Buckner, J., Eggleston, A., \& Schmidt, N. (2006). Social Anxiety and Problematic Alcohol Consumption: The Mediating Role of Drinking Motives and Situations. Behavior Therapy, 37(4), 381-391. doi:10.1016/j. beth.2006.02.007

Buckner, J., Schmidt, N., Lang, A., Small, J., Schlauch, R., \& Lewinsohn, P. (2008). Specificity of social anxiety disorder as a risk factor alcohol and cannabis dependence. Journal of Psychiatric Research, 42(3), 230-239. doi:10.1016/j.jpsychires.2007.01.002

Buckner, J., Tímpano, K., Zvolensky, M., SachEricsson, N., \& Schmidt, N. (2008). Implications of comorbid alcohol dependence among individuals with social anxiety disorder. Depression and Anxiety, 25(12), 10281037. doi:10.1002/da.20442

Buckner, J., \& Turner, J. (2009). Social anxiety disorder as a risk for alcohol use disorders: A prospective examination of parental and peer influences. Drug and Alcohol Dependence, 100, 128-137. doi:10.1016/j.drugalcdep.2008.09.018

Campo-Arias, A., Cogollo, Z., \& Díaz, C. (2008). Comportamientos de riesgo para la salud en adolescentes estudiantes: prevalencia y factores asociados. Salud Uninorte, 24(2), 226-234.

Chassin, L., Pitts, S., \& Prost, J. (2002). Binge drinking trajectories from adolescence to emerging adulthood in a high-risk aample: Predictors and substance abuse outcomes, Journal of Consulting and Clinical Psychology, 20(1), 67-78. doi:10.1037//0022-006X.70.1.67

Clark, D., \& Wells A. (1995). A cognitive model of social phobia. In: Heimberg R, Liebowitz M. y Hope D, Schneier F. editors. Social phobia: diagnosis, assessment and treatment. New York: Guilford.

Conger, J. (1956). Alcoholism: Theory, problem and challenge. Reinforcement theory and the dynamics of alcoholism. Quarterly Journals on Studies of Alcohol, 17(2), 296 -305.

Consejo Nacional contra Adicciones, Instituto Nacional de Psiquiatría Ramón de la Fuente, Instituto Nacional de Salud Pública, Fundación Gonzalo Río Arronte IAP. (2008). Encuesta Nacional de Adicciones 2008. Recuperado de: http://www.conadic.salud.gob. mx/pdfs/ena08/ENA08_NACIONAL.pdf

Crum, R., \& Pratt, L. (2001). Risk of Heavy Drinking and Alcohol Use Disorders in Social Phobia: A prospective Analysis. American Journal of Psychiatry, 158, 1693-1700. doi:10.1176/appi.ajp.158.10.1693

del Bosque, J., Fernández, C., Fuentes, A., Díaz, D., Espínola, M., González, ... Zinser, J. 
(2012). Hacia una mejor respuesta ante el problema del abuso de bebidas con alcohol: el papel del sector salud. Salud Mental, 35, 165-174.

Díaz, R., Díaz, A., Hernández-Ávila, C., Fernández, H., Solís, C., \& Narro, J. (2009). El consumo riesgoso y dañino de alcohol y sus factores predictivos en adolescentes del bachillerato. Salud Mental, 32, 447-458.

Eggleston, A., Woolaway-Bickel, K., \& Schmidt, N. (2004). Social Anxiety and alcohol use: evaluation of the moderating and mediating effects of alcohol expectancies. Journal of Anxiety Disorders, 18(1), 33-49. doi:10.1016/j. janxdis.2003.07.005

Ellis, B., Del Giudice, M., Dishion, T., Figueredo, A., Gray, P., Griskevicius, V., ...Wilson, D. (2012). The Evolutionary Basis of Risky Adolescent Behavior: Implications for Science, Policy, and Practice. Developmental Psychology, 48(3) ,598-623. doi:10.1037/a0026220

Ezpeleta, L. (2005). Factores de Riesgo en Psicopatología del Desarrollo. Barcelona: Masson.

Fantín, M., \& Barbenza, C. (2007). Nivel socioeconómico y consumo de sustancias en una muestra de adolescentes escolarizados de San Luis Argentina. Fundamentos en Humanidades, Universidad Nacional de San Luis Argentina. VIII, 1,15, 133-145.

Flores, L. (2007). Psicología Social de la Salud. Colombia: El Manual Moderno.

García-López, L., Piqueras, J., Díaz-Castela, M., \& Inglés, C. (2008). Trastorno de ansiedad social en la infancia y la adolescencia: Estado actual, avances recientes y líneas futuras. Behavioral Psychology, 16(3), 501-533.

Guzmán, F., Pedrão, L., Rodríguez, L., López, K., \& Esparza, S. (2007). Trastornos por consumo de alcohol (AUDIT) en adolescentes y jóvenes marginales de bandas juveniles de México. Esc Anna Nery Rev Enferm, 11(4), 611-618.

Ham, L., \& Hope, D. (2005). Incorporating social anxiety into a model of college student problematic drinking. Addictive Behaviors, 30(1), 127-150. doi:10.1016/j.addbeh.2004.04.018

Higgins, R., \& Marlatt, G. (1975). Fear of interpersonal evaluation as a determinant of al- cohol consumption in male social drinkers. Journal of Abnormal Psychology, 84(6), 644651. doi:10.1037/0021-843X.84.6.644

Inglés, C., La Greca, A., Marzo, J., García-López, L., \& García-Fernández, J. (2010). Social Anxiety Scale for Adolescents: Factorial invariance and latent mean differences across gender and age in Spanish adolescents. Journal of Anxiety Disorders, 24(8), 847-855. doi:10.1016/j.janxdis.2010.06.007

Instituto Nacional de Estadística, Geografía e Informática. (2004). Encuesta Nacional de Adicciones 2002. Recuperado de http:// www.inegi.gob.mx/prod_serv/contenidos/espanol/bvinegi/productos/continuas/sociales/ salud/2004/ena02.pdf

Instituto Nacional de Psiquiatría Ramón de la Fuente Muñiz, Instituto Nacional de Salud Pública. (2012). Encuesta Nacional de Adicciones 2011: Reporte de Alcohol.

Recuperado de http://encuestas.insp.mx/ena/ ena2011/ENA2011_alcohol.pdf

Khantzian, E. (1987). The self-medication hypothesis of addictive disorders: Focus on heroin and cocaine dependence. American Journal Psychiatry, 142: 1259-1264. doi. org/10.1007/978-1-4613-1837-8_7

Kashdan, T., Collins, R., \& Elhai, J. (2006). Social Anxiety and Positive Outcome Expectancies on Risk-Taking Behaviors. Cognitive Therapy Research, 30, 749-761. doi:10.1007/ s10608-006-9017-x

Kashdan, T., \& Herbert, J. (2001). Social Anxiety Disorder in Childhood and Adolescence: Current Status and Future Directions. Clinical Child and Family Psychology Review, 4(1), 37-62. doi:10.1023/A:1009576610507

Kashdan, T., \& McKnight, E. (2010). The Darker Side of Social Anxiety: When Aggressive Impulsivity Prevails Over Shy Inhibition. Current Directions in Psychological Science, 19(1), 47-50. doi:10.1177/0963721409359280

Kashdan, T., \& Roberts, J. (2004). Social Anxiety's Impact on Affect, Curiosity, and social Self-Efficacy during a High Self-Focus Social Threat Situation. Cognitive Therapy and Research, 28(1), 119-141.

Kashdan, T., \& Savostyanova, A. (2011). Capturing the Biases of Socially Anxious People by 
Addressing Partner Effects and Situational Parameters. Behavior Therapy, 42(2), 211-223. doi:10.1016/j.beth.2010.07.004

Kerlinger, F., \& Lee, H. (2002). Investigación del Comportamiento. Métodos de Investigación en Ciencias Sociales. México: McGrawHill. $4^{\mathrm{a}}$ edición.

Kessler, C. (2003). The impairments caused by social phobia in the general population: implications for intervention. Acta Psychiatrica Scandinavica, 108, suppl: 19-27.

doi:10.1034/j.1600-0447.108.s417.2.x

Kessler, R., McGonagle, K., Zhao, S., Nelson, C., Hughes, M., Eshleman, S., Wittchen, H., \& Kendler, K. (1994). Lifetimeand 12-Month Prevalence of DSM-III-R Psychiatric Disorders in the United StatesResults From the National Comorbidity Survey, Formerly Archives of Gender Psychiatry, 51(1), 8-9.

doi:10.1001/archpsyc.1994.03950010008002

Kushner, M., Sher, K., \& Beitman, B. (1990). The relation between alcohol problems and the anxiety disorders. The American Journal of Psychiatry, 147(6), 685-695.

Kushner, M., Sher, K., \& Erickson, D. (1999). Prospective Analysis of the Relation Between DSM-III Anxiety Disorders and Alcohol Use Disorders, Am J Psychiatry, 156, 723-732. La Greca, A., \& López, N. (1998). Social Anxiety Among Adolescents: Linkages with Peer Relations and Friendsheeps. Journal of Abnormal Child Psychology, 26(2), 83-94.

doi:10.1023/A:1022684520514

Lucio, E., Gómez, H., Morales, B., \& Pérez, M., (2009). Uso del AUDIT y el DAST-10 para la identificación de abuso de sustancias psicoactivas y alcohol en adolescentes. Revista Colombiana de Psicología, 18(1), 9-17.

Medina-Mora, M., García-Tellez, I., Cortina, D., Orozco, R., Robles, R., Vázquez-Pérez, L., Real, T., \& Chisholm, D. (2010). Estudio de costo-efectividad de intervenciones para prevenir el abuso de alcohol en México. Salud Mental, 33, 373-378.

Miers, A., Blöte, A., Bokhorst, C., \& Westenberg, M. (2009). Negative self- evaluations and the relation to performance level in socially anxious children and adolescents. Behaviour Research and Therapy, 47(12), 1043-
1049. doi:10.1016/j.brat.2009.07.017

Nuño-Gutierrez, B., Alvarez-Nemegyei, J., Madrigal-de León, E., \& Rasmussen-Cruz, B. (2005). Prevalencia y factores asociados al consumo de tabaco en adolescentes de una preparatoria de Guadalajara, Jalisco, México. Salud Mental, 28(5), 64-70.

Olivares, J., Ruiz, X., Hidalgo, M., García-López, L., Hernández, M., Rosa, A., \& Piqueras, J. (2005). Social Anxiety Scale for Adolescents (SAS-A): Psychometric properties in a Spanish-speaking population. International Journal of Clinical and Health Psychology, 5(1), 85-97.

Pérez, M., \& Lucio-Gómez, E. (2010). Construcción de un modelo de riesgo en el consumo de alcohol y otras sustancias ilícitas en adolescentes estudiantes de bachillerato, Health and Adicctions, 10, 77-96.

Pons, J., \& Buelga, S. (2011). Factores Asociados al Consumo Juvenil de Alcohol: Una Revisión desde la Perspectiva Psicosocial y Ecológica. Intervención Psicosocial: Revista sobre Igualdad y Calidad de Vida, 20(1), 75-94. doi:10.5093/in2011v20n1a7

Rao, P., Beidel, D., Turner, S., Ammerman, T., Crosby, L., \& Sallee, F. (2007). Social anxiety disorder in childhood and adolescence: Descriptive psychopathology. Behaviour Research and Therapy, 45, 1181-1191. doi:10.1016/j. brat.2006.07.015

Rapee, M., \& Heimberg, R. (1997). A cognitive behavioral model of anxiety in social phobia. Behav. Res. \& Ther. 35(8), 741-756. doi:10.1016/S0005-7967(97)00022-3

Robinson, J., Sareen, J., Cox, B., \& Bolton, J. (2009). Self-medication of anxiety disorders with alcohol and drugs: Results from a nationally representative simple. Journal of Anxiety Disorders, 23, 38-45. doi:10.1016/j. janxdis.2008.03.013

Saunders, J., Aasland, O., Babor, T., De la Fuente, J., \& Grant, M. (1993). Development of the Alcohol Use Disorders Identification Test (AUDIT): WHO collaborative Project on Early Detection of Persons with Harmful Alcohol Consumption-II. Addiction, 88, 791804. doi:10.1111/j.1360-0443.1993.tb02093.x

Schneier, F., Foose, T., Hasin, D., Heimberg, R., 
Liu, S., Grant, B., \& Blanco, C. (2010). Social Anxiety Disorder and Alcohol Use Disorder Comorbidity in the National Epidemiologic Survey on Alcohol and Related Conditions. Psychol Med, 40(6) ,977-988. doi:10.1017/ S0033291709991231

Sher, K., \& Levenson, R. (1982). Risk for alcoholism and individual differences in the stress-response-dampening effect of alcohol. Journal of Abnormal Psychology, 91, 350367. doi:10.1037/0021-843X.91.5.350

Schultz, L., \& Heimberg, R. (2008). Attentional focus in social anxiety disorder: Potential for interactive process. Clinical Psychology Review, 28, 1206-1221. doi:10.1016/j. cpr.2008.04.003

Stice, E. (2002). Risk and Maintenance Factors for Eating Pathology: A Meta-Analytic Reewiew. Psychologycal Bulletin, 128(5), 825-824. doi:10.1037//0033-2909.128.5.825

Stoyanova, M., \& Hope, D. (2012). Gender, gender roles, and anxiety: Perceived confirmability of self report, behavioral avoidance, and physiological reactivity. Journal of anxiety, 26(1), 206-214. doi:10.1016/j.janxdis.2011.11.006

Thomas, S., Randall, C., \& Carrigan, M. (2003). Drinking to Cope in Socially Anxious Individuals: A Controlled Study. Alcoholism: Clinical and Experimental Research, 27(12), 1937-1943. doi:0.1097/01.ALC.0000100942.30743.8C

Thomas, S., Randall, P., Book, S., \& Randall, C. (2008). A complex Relationship Between Co-ocurring Social Anxiety and Alcohol Use Disorders: What Effect Does Treating Social Anxiety Have on Drinking?. Alcoholism: Clinical and Experimental Research, 32(1), 77-85. doi:10.1111/j.1530-0277.2007.00546.x

Turk, C., Lerner, J., Heimberg, R., \& Rapee, R. (2001). An integrated cognitive-behavioral model of social anxiety. In S. G. Hofmann, \& P. M. DiBartolo (Eds.), From social anxiety to social phobia: Multiple perspectives (pp. 281-303). Needham Heights, MA: Allyn \& Bacon.

Villatoro, J., Gaytán, F., Moreno, M., Gutiérrez, M., Oliva, N., Bretón, M., ..., Blanco, C. (2011). Tendencias del Uso de Drogas en la Ciudad de México: Encuesta de Estudiantes del 2009. Salud Mental, 34(81), 94.

Villatoro, J., Gutiérrez, M., Quiroz, N., Moreno, M., Gaytán, L., Gaytán, F., \& Amado, N. (2009). Encuesta de estudiantes de la Ciudad de México 2006. Prevalencias y evolución del consumo de drogas. Salud Mental, 32(4), 287-297.

Wancata, J., Fridl, M., \& Friedrich, F. (2009). Social Phobia: Epidemiology and Health Care. Psychiatria Danubina, 21(4), 520-524.

Wissink, I., Dekovic, M., \& Meijer, A. (2009). Adolescent Friendship Relations and Development Outcomes. Ethnic and Gender Differences. Journal of Early Adolescence, 29(3), 405-425. doi:10.1177/0272431608322945

Xu, Y., Schneier, K., Heimberg, R., Princisvalle, K., Leibowitz, M., Wang, S., \& Blanco, C. (2012). Gender differences in social anxiety disorder: Results from the national epidemiologic sample on alcohol and related conditions, Journal of Anxiety Disorders, 26(1), 12-19. doi:10.1016/j.janxdis.2011.08.006

Zimmermann, P., Wittchen, H., Höfler, M., Pfister, H., Kessler, R., \& Lieb, R. (2003). Primary anxiety disorders and the development of subsequent alcohol use disorders: a 4-year community study of adolescents and young adults. Psychological Medicine, 33, 1211-1222. doi:10.1017/S0033291703008158

Zubeidat, I., Sierra, J., \& Fernández, A. (2006). Ansiedad y fobia social: comorbilidad con otros trastornos psicopatológicos en población adulta e infanto-juvenil. Cuadernos de Medicina Psicosomática y Psiquiatría de Enlace, 79(80), 9-21.

Auto-referencias de autor: 0

Auto-referencias de la revista: 0 